\title{
Fitoextracción De Plomo, Zinc y Cadmio de Relaves Mineros Utilizando Helianthus annuus L. (Girasol)
}

Phytoextraction of Lead, Zinc and Cadmium of Mining Tailings Using Helianthus annuus L. (Sunflower)

Katherine Y. Lizarbe Asmat ${ }^{1}$, Yaslin S. Rivera López ${ }^{1}$, Jorge L. Mendoza Bobadilla ${ }^{1}$, Manuel Vera Herrera ${ }^{1}$, Ronald F. Rodríguez Espinoza ${ }^{2}$

\section{RESUMEN}

Objetivo: Evaluar el crecimiento de Helianthus annuus L. (girasol), especie bioacumuladora de metales pesados, mediante la adición de acondicionadores orgánicos e inorgánicos al relave minero artesanal, para la fitoextracción de plomo, zinc y cadmio. Métodos: Se probaron cuatro tratamientos de diferentes proporciones relave - acondicionador (100-0\%, $75-25 \%, 50-50 \%, 25-75 \%$ respectivamente), con seis repeticiones cada uno, para periodos de tiempo de 30,60 y 90 días y mediante dos métodos de siembra (directa y trasplante). Se realizaron análisis fisicoquímicos a los tratamientos ( $\mathrm{pH}$, conductividad eléctrica, materia orgánica, fósforo disponible y potasio disponible), y se determinó la concentración de $\mathrm{Pb}, \mathrm{Zn}$ y $\mathrm{Cd}$ en el relave y en los órganos vegetales del girasol. Resultados: Los resultados muestran que el girasol logró crecer en los tratamientos con adición de acondicionador y acumular en sus tejidos $\mathrm{Pb}, \mathrm{Zn}$ y $\mathrm{Cd}$; viéndose afectada la concentración de estos metales, con respecto al tiempo, por el contenido de materia orgánica de los tratamientos. Conclusiones: El método de siembra directa fue el que logró mayor concentración de $\mathrm{Pb}, \mathrm{Zn}$ y Cd, siendo la raíz el órgano de mayor bioacumulación de estos metales. Asimismo, se observó que los mejores resultados obtenidos se encuentran en el tratamiento 2 (75\% de relave y $25 \%$ de acondicionador).

Palabras clave: Helianthus annuus L. (girasol), relave minero, fitoextracción, acondicionador.

\section{ABSTRACT}

Objective: Evaluate the growth of Helianthus annuus L. (sunflower), bioaccumulative species of heavy metals, by adding inorganic and organic conditioners to artisanal mining tailings for the phytoextraction of lead, zinc and cadmium. Methods: Four treatments were tested of different proportions tailings - conditioner (100 - 0\%, $75-25 \%, 50-50 \%, 25-75 \%$, respectively), with 6 replicates each, for periods of 30,60 and 90 days by two seeding methods (direct and transplantation). Physicochemical analysis were performed at the treatments $(\mathrm{pH}$, electrical conductivity, organic matter, available phosphorus and available potassium), and determined the concentration of $\mathrm{Pb}, \mathrm{Zn}$ and $\mathrm{Cd}$ in the tailings and vegetables organs of sunflower. Results: The results show that the sunflowers achieved to grow in treatments with added conditioner and accumulate in their tissues $\mathrm{Pb}$, $\mathrm{Zn}$ and $\mathrm{Cd}$; being affected the concentration of these metals, with respect to time, by the organic matter content of the treatments. Conclusions: The method of direct seeding is the greatest amount of $\mathrm{Pb}, \mathrm{Zn}$ and $\mathrm{Cd}$ concentrated, being the root the organ the highest bioaccumulation of these metals. Also, was observed that the best results are obtained in treatment 2 (75\% of tailings and $25 \%$ conditioner).

Keywords: Helianthus annuus L. (sunflower), mining tailings, phytoextraction, conditioner. 


\section{INTRODUCCIÓN}

La minería artesanal mantiene una presencia significativa en la producción minera del Perú, principalmente en la producción de oro. "Las operaciones artesanales se encuentran en áreas que han sido explotadas de manera tradicional, tal como algunas zonas en Puno, en donde la explotación de estos yacimientos data desde la época Colonial. La minería artesanal también se practica en minas que han sido abandonadas por las empresas, tal es el caso de las minas de La Libertad, que por problemas de rentabilidad han tenido que ser cerradas" (Kuramoto, 2001).

"En la actualidad en la Región La Libertad, tenemos grandes cantidades de pasivos ambientales (relaves mineros) generados por la minería informal y artesanal, que se dan en las concesiones mineras ubicadas en las provincias de Pataz, Sánchez Carrión, Otuzco, Gran Chimú y otras". La potencialidad del daño ambiental es significativa para las áreas agrícolas que se encuentran en las partes bajas de las zonas de donde extraen el mineral (Gobierno Regional de La Libertad, 2006).

El proceso de cianuración es usado frecuentemente para la lixiviación de minerales de oro y plata. Por lo que Logsdon, Hagelstein y Mudder (2001), afirman que "Los relaves mineros producidos mediante el método de cianuración contienen sustancias tóxicas como el arsénico, plomo, cadmio, cianuro, selenio y zinc, que al tener contacto con el agua y el subsuelo, altera su composición natural.

Los avances tecnológicos para sanear suelos contaminados con metales pesados han conllevado al desarrollo de diversas técnicas, como: fisicoquímicas, de extracción, químicas, de fijación y/o encapsulación, biológicas. Esta última se divide en dos: la biorremediación y la fitorremediación. La primera consiste en eliminar los contaminantes orgánicos de un medio a través de microorganismos, esto se puede realizar "ex situ", mientras que la segunda utiliza especies vegetales como medio de tratamiento. Para llevar a cabo acciones de fitorremediación, existen dos posibilidades: la fitoestabilización o fitoextracción. Estas consisten en la inmovilización de los metales utilizando especies vegetales con capacidad fijadora, o llevando a cabo la extracción de los contaminantes con plantas acumuladoras o hiperacumuladoras, respectivamente (Del
Lobo, 2004).

La fitorremediación, novedosa tecnología, tiene muchas ventajas con respecto a los métodos convencionales de tratamientos de lugares contaminados; puesto que, es una tecnología de bajo costo, posee un impacto regenerativo en lugares en donde se aplica y su capacidad extractiva se mantiene debido al crecimiento vegetal (Harvey et al., 2002).

El éxito de la fitorremediación depende de identificar las especies vegetales que toleren el estrés, que acumulen los metales pesados y produzcan cantidades grandes de biomasa. En general las plantas que acumulan más de 1000 $\mathrm{mg} / \mathrm{kg}$ del $\mathrm{Pb}$, se llaman hiperacumuladoras" (García Chico y Valdivieso, 2008).

Dentro de la familia Asteraceas se encuentra el $H$. annuus $L$. (girasol) que absorbe los metales pesados en mayor cantidad en sus raíces que en sus brotes, si se cosecha la biomasa entera de la planta puede extraer del 10 al $25 \%$ de los metales del suelo. Esta especie absorbe metales pesados en grandes cantidades por lo que se considera una planta hiperacumuladora favorable en la fitoextracción de Cd, Zn, Pb, As y elementos radiactivos (Garcia, Gratão, Azevedo y Arruda, 2006).

El objetivo es evaluar el crecimiento de $H$. annuus $L$. (girasol), especie bioacumuladora de metales pesados, mediante la adición de acondicionadores orgánicos e inorgánicos al relave minero artesanal, para la fitoextracción de plomo, zinc y cadmio.

\section{MATERIALES Y MÉTODOS}

Se utilizó una muestra procedente de la escombrera de relaves minero artesanal, localizado en el Caserío de Zarumilla, Pataz. Se obtuvo las muestras de relave mediante el método de muestreo en zigzag, se excavó hasta $20 \mathrm{~cm}$ de profundidad aproximadamente y se extrajo $6 \mathrm{~kg}$ de submuestras en 20 puntos de muestreos, las cuales se homogenizaron obteniendo una muestra compuesta de $120 \mathrm{~kg}$.

Además, se utilizó una mezcla de material orgánico (humus y musgo) e inorgánico (arena y aserrín) para la preparación de $36 \mathrm{~kg}$ de acondicionador, a partir de la siguiente proporción: $50 \%$ humus $+30 \%$ musgo $+10 \%$ arena $+10 \%$ aserrín . 
Sobre la muestra recolectada se llevó a cabo un total de 4 tratamientos a diferentes proporciones de relave y acondicionador, como se observa en la Tabla 1, se realizó 6 réplicas por tratamiento en bolsas de polietileno de $2 \mathrm{~kg}$ de capacidad.

Tabla 1. Proporciones del relave y acondicionador

\begin{tabular}{cc}
\hline Tratamiento & Descripción \\
\hline T1 & $100 \%$ relave \\
T2 & $75 \%$ relave $+25 \%$ de acondicionador \\
T3 & $50 \%$ relave $+50 \%$ de acondicionador \\
T4 & $25 \%$ relave $+75 \%$ de acondicionador \\
\hline
\end{tabular}

Las semillas de $H$. annuus $L$. fueron desinfectadas por inmersión en una solución de agua con Tiran (fungicida de contacto) por un minuto, para eliminar cualquier bacteria y protegerlas del ataque de hongos patógenos.

La especie tuvo condiciones de crecimiento según los métodos de siembra:

Siembra directa: Se colocaron 2 semillas en cada bolsa con los diferentes tratamientos, las cuales fueron regadas diariamente con el fin de activar el embrión de la planta.

Trasplante: La germinación se realizó en una bandeja de germinación de 72 semillas en donde se colocó 1 semilla, las cuales se mantuvieron húmedas con agua destilada y en condiciones semicontroladas de invernadero hasta su germinación, después de 10 días las plántulas se seleccionaron y trasplantaron a las bolsas con los sustratos preparados para los diferentes tratamientos.

El experimento tuvo una duración de 100 días para asegurar la confiabilidad de los datos; tomando como referencia para la evaluación a 30,60 y 90 días.

Para evaluar el efecto de las propiedades fisicoquímicas de los sustratos en la fitoextracción de $\mathrm{Pb}, \mathrm{Zn}$ y Cd, se realizaron los siguientes análisis fisicoquímicos a cada tratamiento: $\mathrm{pH}$, conductividad eléctrica, materia orgánica, fósforo disponible y potasio disponible.
Se tomaron medidas de longitud de raíz, número de hojas, longitud de tallo y peso seco, de todas las plantas de $\mathrm{H}$. annuus $\mathrm{L}$. correspondientes a los 4 tratamientos para los periodos de 30, 60 y 90 días y fueron comparados con un "Control" (plantas germinadas en suelo no contaminado).

Se usó un espectrofotómetro de absorción atómica (EAA) Avanta PM para cuantificar el contenido de $\mathrm{Pb}, \mathrm{Zn}$ y $\mathrm{Cd}$ en el relave y lo acumulado en la raíz y parte aérea (hojas, tallos y botón floral) de las plantas, las cuales fueron evaluadas a los 30,60 y 90 días.

Para las muestras de plantas de cada tratamiento elegimos 2 plantas de las cuales se separaron las raíces, tallos y hojas. Se tomaron sus masas antes y después de secar en una estufa eléctrica a $105^{\circ} \mathrm{C}$.

Se tomó 0,2 gramos de cada muestra, vertiéndose en fiolas rotuladas de $50 \mathrm{ml}$ de capacidad, se homogenizaron con $6 \mathrm{ml}$ de $\mathrm{HNO}_{3}$ cc y $4 \mathrm{ml}$ de $\mathrm{HCl}$, luego fueron llevadas a digestión ácida por 1 hora utilizando una plancha eléctrica. Cuando el contenido clarificó se aforó con agua destilada hasta completar 50 $\mathrm{ml}$ (relave) y $25 \mathrm{ml}$ (plantas), se filtró y la lectura se realizó en el EAA.

Todos los análisis estadísticos realizados fueron evaluados en el programa SPSS 20.0. Los parámetros de crecimiento y concentración de metales de las plantas dentro de cada tratamiento fueron comparados mediante un análisis de varianza multivariante (MANOVA), con nivel de confianza del $95 \%(p<0,05)$ y $99 \%$ $(p<0,01)$. Mediante el test "post-hoc" prueba de Tukey se realizó una comparación múltiple de medias entre los tratamientos en relación a las concentraciones de $\mathrm{Pb}, \mathrm{Zn}$ y $\mathrm{Cd}$.

\section{RESULTADOS}

Los resultados de los parámetros fisicoquímicos de los tratamientos al inicio del trabajo se muestran en la Tabla 2. La concentración promedio de metales pesados se muestra en la Tabla 3, y la concentración promedio de metales pesados en la materia seca (MS) a $105^{\circ} \mathrm{C}$ en $\mathrm{mg} / \mathrm{kg}$ en los órganos de la planta $H$. annuss $L$. se muestran en la Tabla 4. 
Tabla 2. Parámetros fisicoquímicos de los tratamientos al inicio del trabajo

\begin{tabular}{lrrrr}
\hline & \multicolumn{5}{c}{ Tratamientos } \\
\cline { 2 - 5 } Parámetros Fisicoquímicos & T1 & T2 & T3 & T4 \\
\hline pH & 8,5 & 7,9 & 7,7 & 7,4 \\
Conductividad eléctrica (dS/m) & 5,7 & 4,6 & 5,9 & 6,8 \\
Materia orgánica (\%) & 0,9 & 2,8 & 6,9 & 10,9 \\
Fósoforo disponible (ppm) & 74,0 & 74,0 & 82,0 & 126,0 \\
Potasio disponible (ppm) & 207,0 & 294,0 & 294,0 & 294,0 \\
\hline
\end{tabular}

Fuente: Laboratorio de análisis de suelos, Facultad de Ciencias Agropecuarias UNT.

Tabla 3. Contenido de metales pesados en el relave

\begin{tabular}{llccc}
\hline $\begin{array}{l}\text { Metales } \\
\text { Pesados }\end{array}$ & Unidad & Valores & $\begin{array}{c}\text { Estándares de } \\
\text { Calidad Ambiental } \\
\text { Suelo }\end{array}$ & $\begin{array}{c}\text { Directrices de } \\
\text { Calidad } \\
\text { Ambiental de } \\
\text { Canadá }\end{array}$ \\
\hline Plomo & $\mathrm{mg} / \mathrm{kg} \mathrm{MS}$ & 3178,29 & 1200 & 600 \\
Zinc & $\mathrm{mg} / \mathrm{kg} \mathrm{MS}$ & 1618,75 & No establece & 360 \\
Cadmio & $\mathrm{mg} / \mathrm{kg} \mathrm{MS}$ & 17,38 & 22 & 22 \\
\hline
\end{tabular}

(1)D.S. 002 - 2013 MINAN: Aprueban Estándares de Calidad Ambiental (ECA) para Suelo.

(2)Guía Canadiense para la Calidad del Suelo para la Protección de la Salud Ambiental y Humana.

Tabla 4. Concentración promedio de metales pesados (mg/kg MS) en los órganos de la planta Helianthus annuss $L$.

\begin{tabular}{|c|c|c|c|c|c|c|c|c|c|c|c|}
\hline \multirow{3}{*}{$\begin{array}{c}\text { Metales } \\
\text { pesados } \\
\text { (mg/kg MS)) }\end{array}$} & \multirow{3}{*}{ Órganos } & \multirow{3}{*}{$\begin{array}{l}\text { Metodo } \\
\text { de } \\
\text { siembra }\end{array}$} & \multicolumn{9}{|c|}{ Tratamientos } \\
\hline & & & \multicolumn{3}{|c|}{ T2 } & \multicolumn{3}{|c|}{ T3 } & \multicolumn{3}{|c|}{ T4 } \\
\hline & & & 30 días & 60 días & 90 días & 30 días & 60 días & 90 dias & 30 días & 60 días & 90 dias \\
\hline \multirow{4}{*}{ Plomo } & \multirow{2}{*}{$\begin{array}{l}\text { Parte } \\
\text { Aérea }\end{array}$} & $\begin{array}{l}\text { Siembra } \\
\text { directa }\end{array}$ & 495,97 & 512,36 & 586,63 & 445,70 & 254,78 & 219,32 & 194,64 & 154,72 & 97,45 \\
\hline & & Transplante & 113,44 & 197,30 & 241,51 & 167,56 & 151,74 & 127,95 & 134,75 & 112,45 & 96,32 \\
\hline & \multirow{2}{*}{ Raíz } & $\begin{array}{l}\text { Siembra } \\
\text { directa }\end{array}$ & 414443 & 4656,35 & 4937,62 & 3923,92 & 2811,78 & 2256,71 & 818,72 & 745,21 & 622,46 \\
\hline & & Transplante & 2486,66 & 2793,81 & 2962,57 & 2354,35 & 1687,07 & 1415,60 & 425,63 & 375,84 & 364,52 \\
\hline \multirow{4}{*}{ Zinc } & \multirow{2}{*}{$\begin{array}{l}\text { Parte } \\
\text { Aérea }\end{array}$} & $\begin{array}{c}\text { Siembra } \\
\text { directa }\end{array}$ & 236,26 & 452,13 & 544,01 & 283,33 & 211,45 & 192,98 & 183,69 & 123,24 & 102,47 \\
\hline & & Transplante & 165,38 & 316,49 & 380,81 & 156,83 & 138,02 & 110,79 & 128,58 & 96,27 & 81,73 \\
\hline & \multirow{2}{*}{ Raíz } & $\begin{array}{c}\text { Siembra } \\
\text { directa }\end{array}$ & 130,26 & 1188,63 & 1206,32 & 1093,62 & 754,42 & 675,06 & 371,19 & 298,24 & 212,62 \\
\hline & & Transplante & 687,69 & 781,14 & 856,25 & 614,42 & 394,12 & 223,36 & 301,24 & 254072 & 212,26 \\
\hline \multirow{4}{*}{ Cadmio } & \multirow{2}{*}{$\begin{array}{l}\text { Parte } \\
\text { Aérea }\end{array}$} & $\begin{array}{c}\text { Siembra } \\
\text { directa }\end{array}$ & 0,9326 & 1,6846 & 1,9879 & 0,5590 & 0,3874 & 0,3655 & 0,2963 & 0,2614 & 0,1988 \\
\hline & & Transplante & 0,5128 & 0,9522 & 1,2215 & 0,3913 & 0,3311 & 0,3114 & 0,1124 & 0,1045 & 0,0987 \\
\hline & \multirow[t]{2}{*}{ Raíz } & $\begin{array}{c}\text { Siembra } \\
\text { directa }\end{array}$ & 5,9382 & 5,9548 & 6,0178 & 46404 & 4,5874 & 4,3815 & 2,6645 & 2,3585 & 2,3237 \\
\hline & & Transplante & 3,8567 & 4,0084 & 4,2546 & 2,9828 & 2,8912 & 2,8717 & 1,7423 & 1,2741 & 1,0204 \\
\hline
\end{tabular}

\section{DISCUSIÓN}

La Tabla 3, muestra la concentración de metales pesados presentes en el relave de estudio, el $\mathrm{Pb}(3178,29 \mathrm{mg} \mathrm{Pb} / \mathrm{kg})$ supera un $265 \%$ los valores establecidos en el Estándar de Calidad de Suelo (ECA-suelos) y $530 \%$ las Directrices de Calidad Ambiental de Canadá (EQG), en el caso del Zn (1618,75 mg Zn/kg), se sabe que es esencial en la nutrición vegetal a nivel de trazas; sin embargo, el valor que reporta es $450 \%$ mayor en comparación al EQG, el Cd (17,38 mg Cd/kg) no supera los umbrales de tolerancia ni de ECA-suelos ni de EQG. Estos valores son indicativos de que el material utilizado como sustrato es altamente contaminado en $\mathrm{Pb}$ y $\mathrm{Zn}$. 
En la Tabla 4 se presentan los resultados de la concentración de $\mathrm{Pb}$ en parte aérea y raíz respectivamente. En el cual se observa que la mayor acumulación se presentó en las raíces, alcanzando la máxima concentración en T2 a 90 días para el método de siembra directa con $4937,62 \mathrm{mg} \mathrm{Pb} / \mathrm{kg}$ y la translocación hacia la parte aérea de $586,63 \mathrm{mg} \mathrm{Pb} / \mathrm{kg}$, esta poca movilidad del metal a través de la planta es debido a que se mantiene adherido a la pared celular de las raíces y, en menor cantidad se transporta hacia las partes aéreas, explicando así porque es común encontrarlo en las raíces (Zúñiga, 1999); lo cual, concuerda con los resultados de García, Chico y Valdiviezo (2008) y Hernández (2006) acerca de que la mayor concentración de $\mathrm{Pb}$ se encuentra en las raíces. También se observa que la concentración en T2 en parte aérea, incrementa de manera pasiva con respecto al tiempo en comparación con el $\mathrm{Zn}$ y $\mathrm{Cd}$ que tuvieron un incremento más pronunciado; caso contrario sucede en T3 y T4 la concentración tiende a disminuir, ya que estos tratamientos poseen mayor porcentaje de acondicionador (50\% y $75 \%$ respectivamente), estando directamente relacionado con el alto contenido de materia orgánica $(\mathrm{MO})$, la cual reacciona con los metales formando complejos de organometálicos, que puede inmovilizar o disminuir la fracción biodisponible para las plantas, coincidiendo los resultados con el estudio realizado por Marín Roga, Calderón y Andrea (2008) para las mismas proporciones de enmienda (tierra de hoja comercial).

En el caso del Cadmio las concentraciones son muy pequeñas en comparación a los valores de $\mathrm{Pb}$ y $\mathrm{Zn}$. El Cd tuvo una mayor acumulación en las raíces que en la parte aérea, alcanzando la máxima acumulación en las raíces de 6,0178 $\mathrm{mg} / \mathrm{kg}$ a los 90 días en T2 para siembra directa y la translocación hacia la parte aérea de la planta de la tercera parte, también con el máximo valor de $1,9879 \mathrm{mg} / \mathrm{kg}$. El Cd, similar al $\mathrm{Zn}$, tiene una mayor movilidad que puede desplazar otros iones metálicos e incorporarse rápidamente a los diferentes tejidos de la planta (Hall, 2002). Una vez más se apreció en T3 y T4 el efecto de la MO aportada por el acondicionador, ya que a medida que aumenta el porcentaje de este en los sustratos, la variación se reduce hasta exhibir en T4 la menor concentración de $\mathrm{Cd}$. Esto se debe a la alta afinidad del Cd para formar complejos orgánicos en los sustratos.
De los resultados obtenidos se concluye, que la adición de acondicionador al relave tuvo una implicancia significativa en el crecimiento de $\mathrm{H}$. annuus L. (girasol) y la concentración de $\mathrm{Pb}, \mathrm{Zn}$ y $\mathrm{Cd}$ en sus órganos vegetales, en donde, la raíz fue el órgano vegetal de $H$. annuus $L$. (girasol) con mayor capacidad bioacumuladora de $\mathrm{Pb}, \mathrm{Zn}$ y $\mathrm{Cd}$. En relación a los métodos de siembra aplicados, el método de siembra directa es el mejor método para la fitoextracción de $\mathrm{Pb}, \mathrm{Zn}$ y $\mathrm{Cd}$ ya que la semilla germinó en el medio contaminado a diferencia del método de trasplante, y por último, de los tratamientos preparados, el tratamiento 2 (75\% relave y $25 \%$ acondicionador) presentó los mejores resultados en la fitoextracción de $\mathrm{Pb}, \mathrm{Zn}$ y Cd, a pesar de tener una menor biomasa en comparación a los otros tratamientos (T3 y T4), concentró mayor cantidad de $\mathrm{Pb}, \mathrm{Zn}$ y $\mathrm{Cd}$ en raíz y parte aérea

\section{REFERENCIAS BIBLIOGRÁFICAS}

CCME (Canadian Council of Ministers of the Environment) (2007). Canadian soil quality guidelines for the protection of environmental and human health.

Del Lobo Bedmar, M. C. (2004). Descontaminación de Suelos. Instituto Madrileño de Investigación Agraria y Alimentaria. IMIA. N-II KM, 38.

García, L., Chico Ruiz, J. \& Valdivieso Arenas, G. (2008). Capacidad de Helianthus Annuus L. "Girasol" para acumular Plomo y su efecto en el crecimiento.

Garcia, J. S., Gratão, P. L., Azevedo, R. A., \& Arruda, M. A. Z. (2006). Metal contamination effects on sunflower (Helianthus annuus L.) growth and protein expression in leaves during development. Journal of agricultural and food chemistry, 54(22), 8623-8630.

Gobierno Regional de la Libertad. Dirección Regional de Energía y Minas La Libertad, 2006.

Hall, J. L. (2002). Cellular mechanisms for heavy metal detoxification and tolerance. Journal of experimental botany, 53(366), 1-11. 
Harvey, P. J., Campanella, B. F., Castro, P. M., Harms, H., Lichtfouse, E., Schäffner, A. R., \& Werck-Reichhart, D. (2002). Phytoremediation of polyaromatic hydrocarbons, anilines and phenols. Environmental Science and Pollution Research, 9(1), 29-47.

Hernández, B. (2006). Fitoextracción de metales pesados Cromo, Plomo y Arsénico contenidos en lodos residuales de las lagunas facultativas de la PTAR Covicorti-Trujillo. Tesis para obtener el grado de Ingeniero Químico. Universidad Nacional de Trujillo, Trujillo.

Kuramoto, J. (2001). La minería artesanal e informal en el Perú. Lima: Grupo de Análisis para el Desarrollo (GRADE).

Logsdon, M. J., Hagelstein, K. \& Mudder, T. (2001). El manejo del cianuro en la extracción de oro. International Council on Metals and the Environment.

Marin Roga, I. J. M. S., Calderon, M. J. O. \& Andrea, C. (2008). Estudio del efecto de polypogon australis sobre las propiedades fisicoquimicas de un sustrato de relave minero de la planta Santo Domingo, Paposo, II Region de Chile Calderón (No. 62: 504 628). e-libro, Corp...
Ministerio del Ambiente (2008, Julio, 31). D.S. $N^{\circ}$ 002-2008-MINAM. Aprueba los Estándares nacionales de calidad ambiental para agua. Diario Oficial El Peruano, 377222 - 377227.

Región La Libertad [En línea]. - Dirección Regional de Energía y Minas La Libertad, 2006. - 25 de noviembre de 2012. http://www.regionlalibertad.gob.pe/web/ opciones/pdfs/DR\%20ENERGIA\%20Y\% 20MINAS\%202006.pdf

Zúñiga, F. B. (1999). Introducción al estudio de la contaminación del suelo por metales pesados (Vol. 1). UADY

\section{Correo electrónico:}

katherine_yelitza@hotmail.com

Revisión de pares:

Recibido: 03-10-2016

Aceptado: 21-12-2016 